\title{
Microcontroller Based Code Locking System with Alarm
}

\author{
Diarah Reuben Samuel, OsuekeChristian.O, Egbune Dickson \\ (Electrical and Information Engineering, College of Science and Engineering/Landmark University Omu-Aran, \\ Nigeria) \\ (Mechanical Engineering, College of Science and Engineering/Landmark University Omu-Aran, Nigeria \\ (Electrical and Information Engineering, College of Science and Engineering/Landmark University Omu-Aran, \\ Nigeria)
}

\begin{abstract}
The need for strict security measures has been necessary since the beginning of time. Access to certain places and items need strict restrictions to only the privileged few. This restricted zone can vary from strong holds and safes in financial institutions to doors leading to restricted areas. The various innovations in security access system include; code based lock, keycard lock, thumb print scan, retina scan. They come in handy in security systems. Code based locking system is best suited in most applications because of its simplicity and reliability. Since the code based locking system is always resident in the area to be protected, there are fewer chances of security breaches unlike the keycard lock system in which the access card can fall into unauthorized hands. Furthermore, in the issue of maintenance, the access code can easily be changed at will with lesser cost unlike in the case of keycard system where a new set of access cards are required. The design and operational principles of a microcontroller based code locking system is the subject of the thesis.The project is made of a keypad unit for entering the access code, a dynamic display unit that displays different messages at specific time, a door controller section made up of a H-bridge driver IC that controls the movement of the motor attached to the door and an alarm system that triggers when the conditions are bridged. The whole system is controlled by an 8051 based microcontroller (89s51).
\end{abstract}

Keywords :Debugging,Hardware, Microcontroller, programming, software.

\section{Introduction}

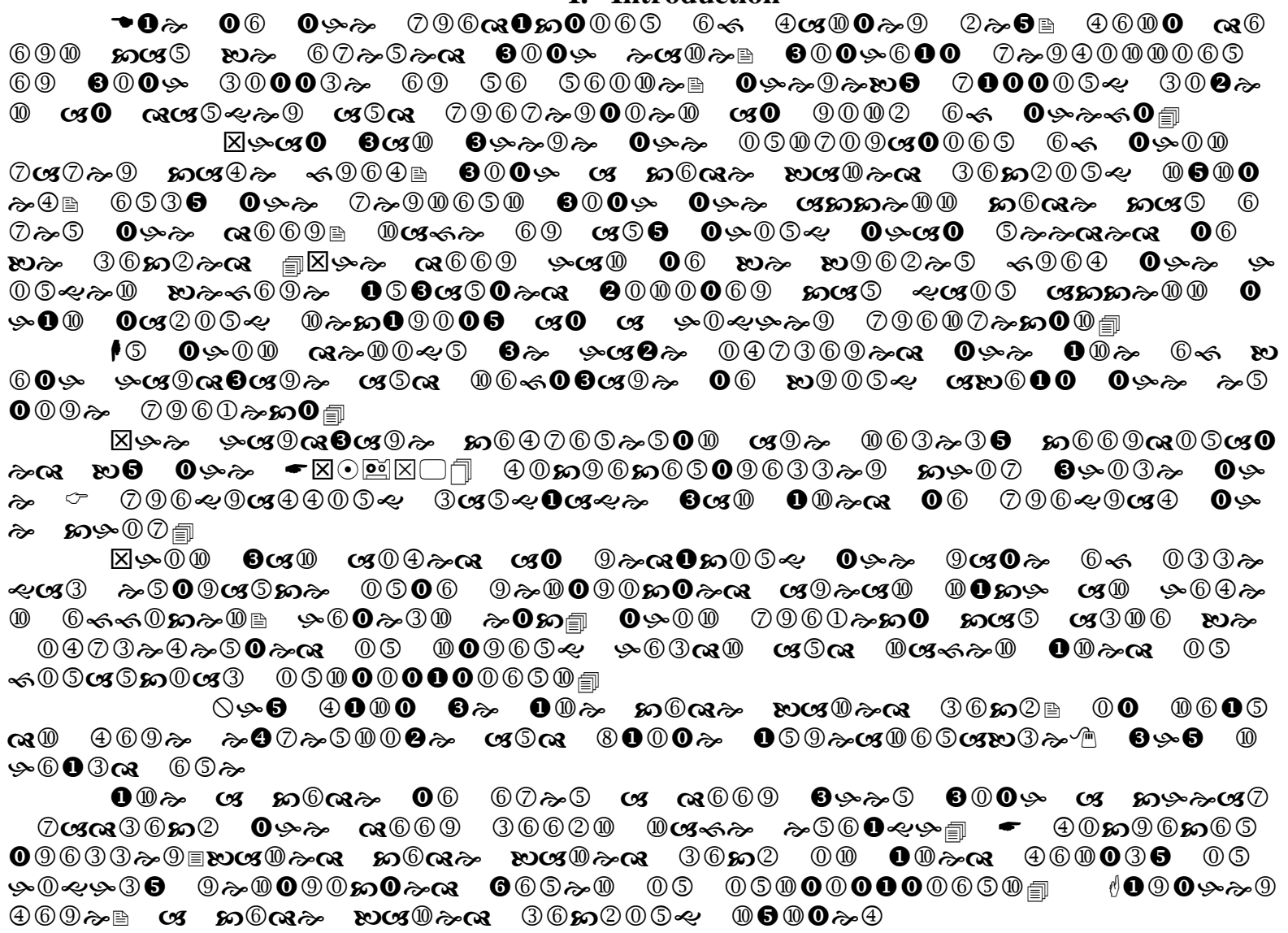




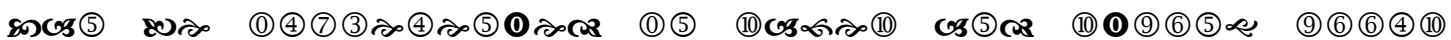

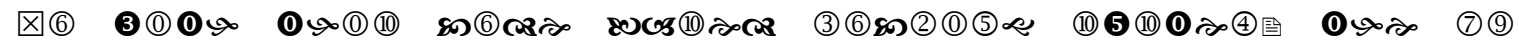

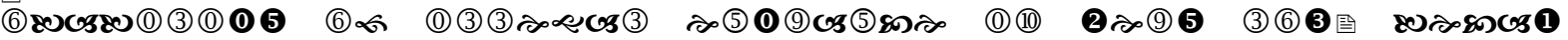

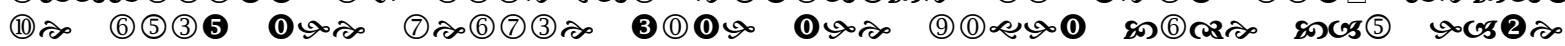

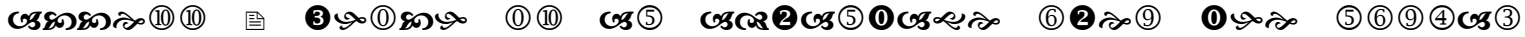
(3) (6) (2) (11) (6) (9) (2) $2(5$ (10)

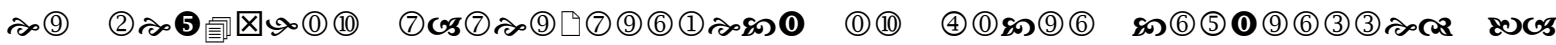
(10) $2 \times 2$

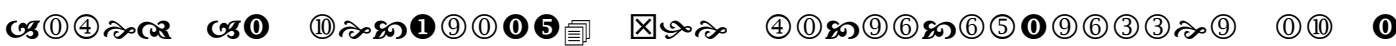

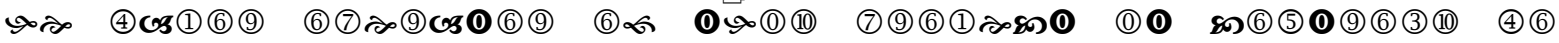
(11) 0 (7) $\cos (9) \quad$ (6) 0 Os उमr(9) sny (1)

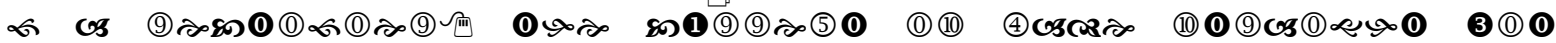

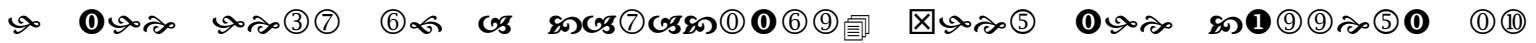

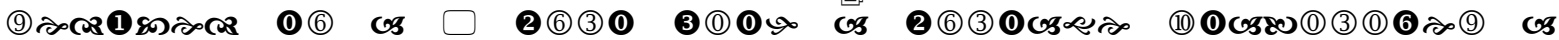

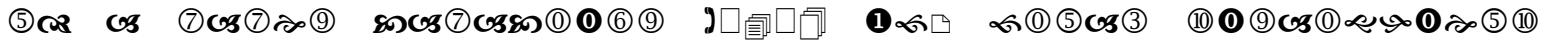
0var $\sin (9)$ (9)

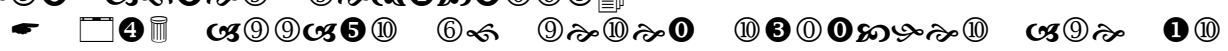

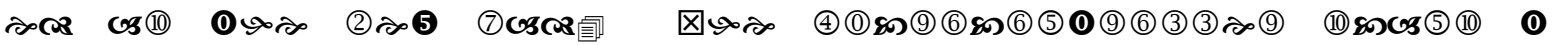
प्र(9) (6) (1)

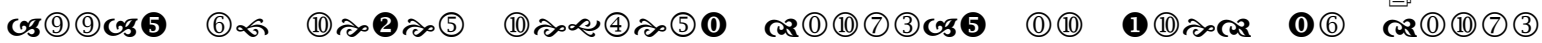

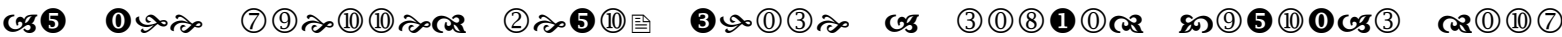

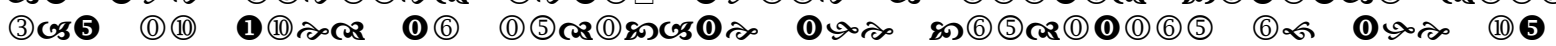

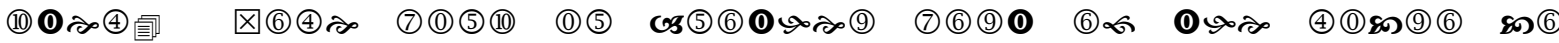
(5) 0 (9) (6) (3) (3) मे (9) 3 (1) (3) (3) घur

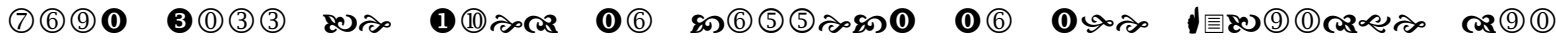

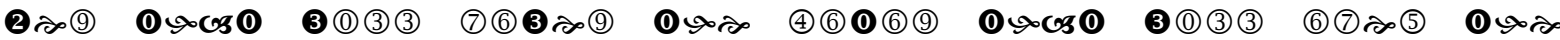

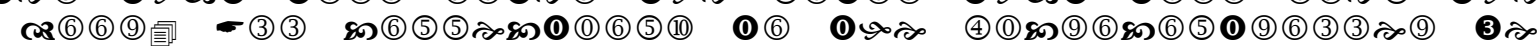

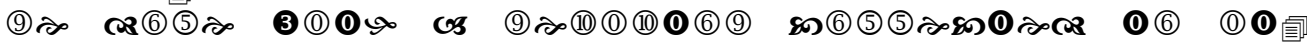

问甸问 $\checkmark$ (9)(6) (1)

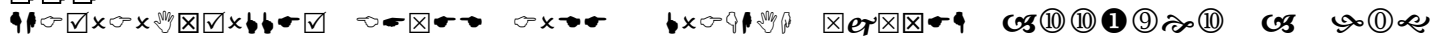

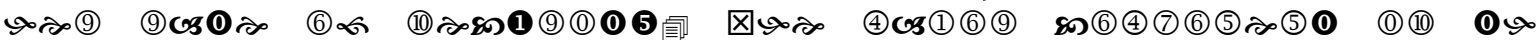

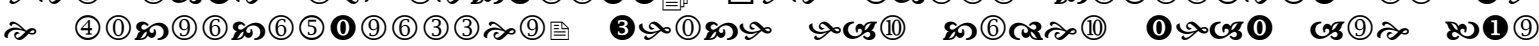

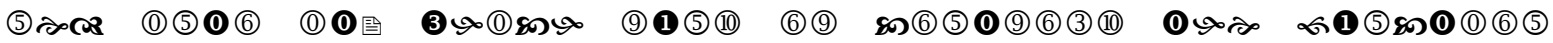

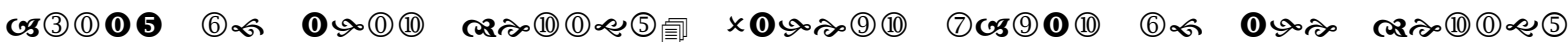
$\cos (9) 2$

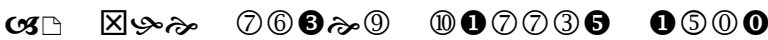

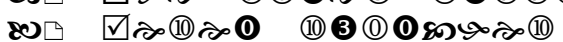

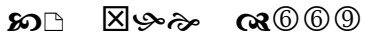

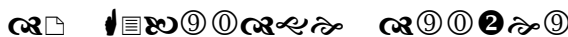

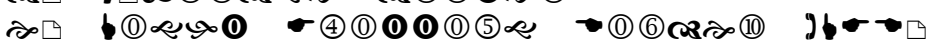

a b

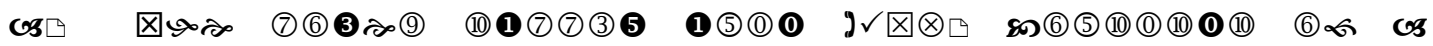

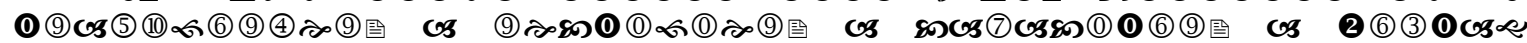

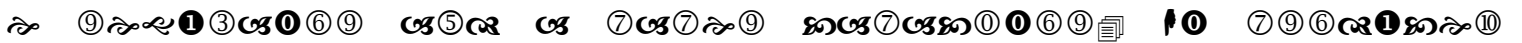
\begin{tabular}{ll}
2 \\
\hline$y$
\end{tabular}

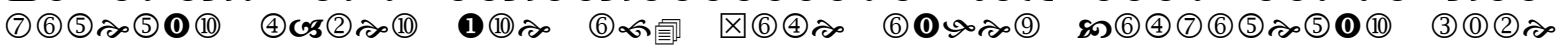

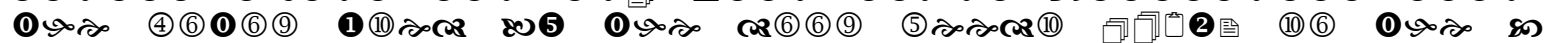
(6) (5) (5) $2 \sin ($ (1) (6) (5) (9)

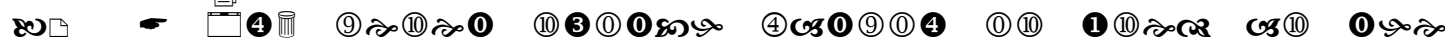
(2) $250 \cos \cos$

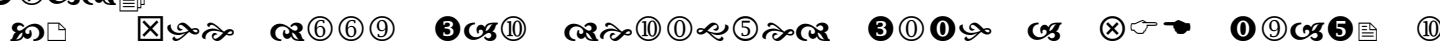

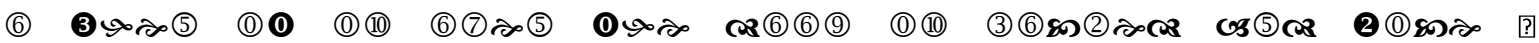

(2) (9) (10) 03

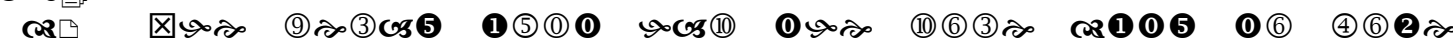

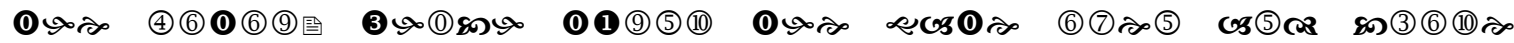

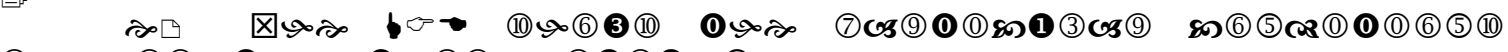

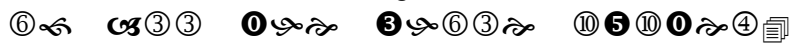

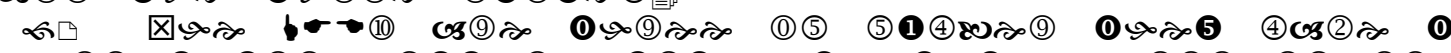

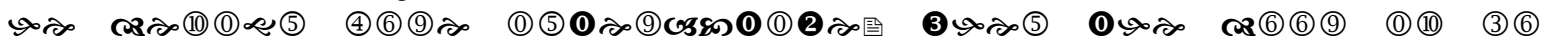




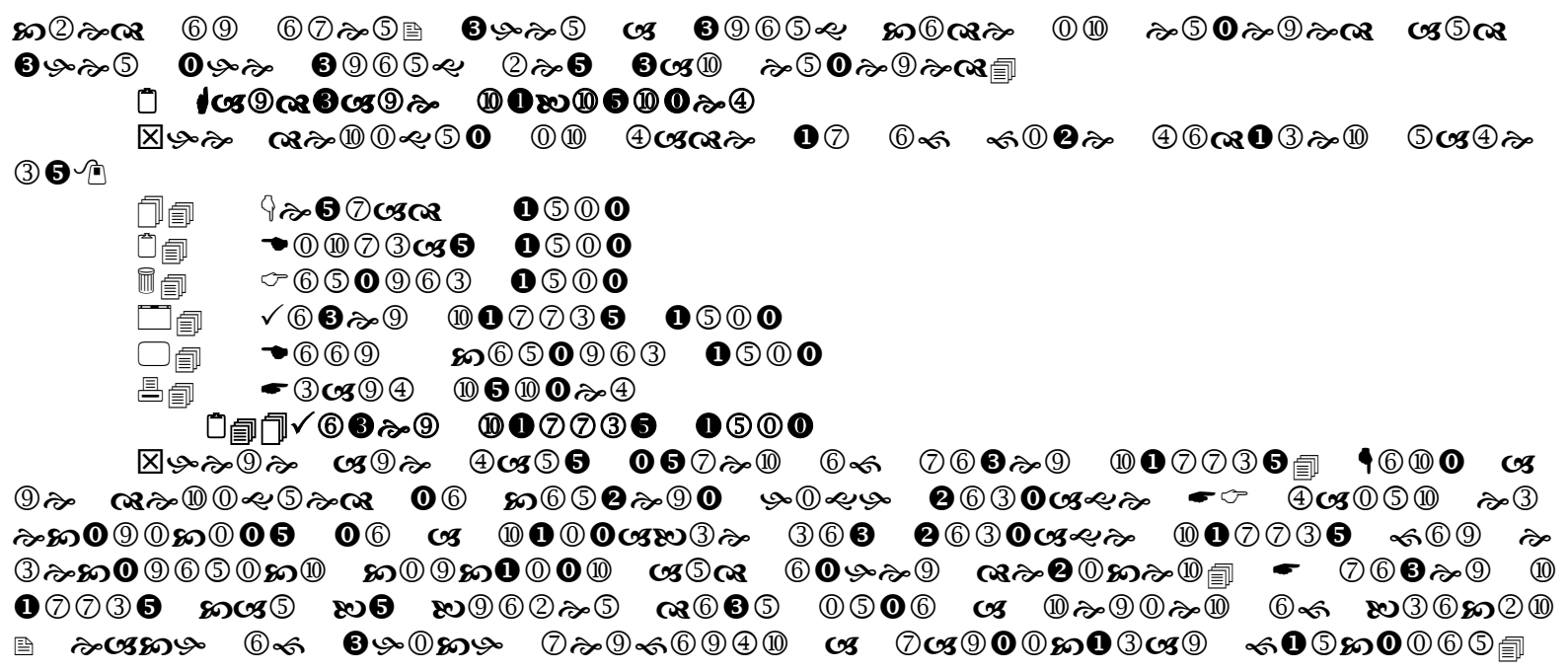

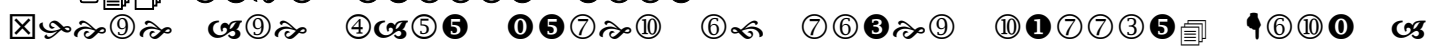
(9)

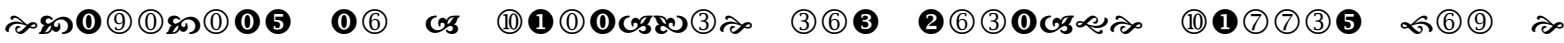

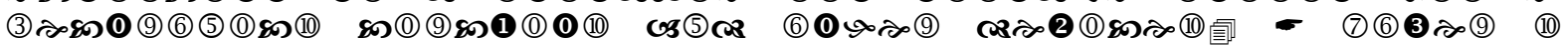

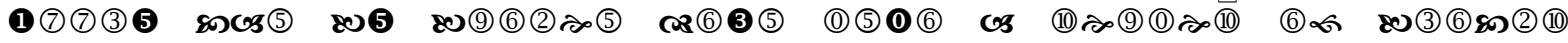

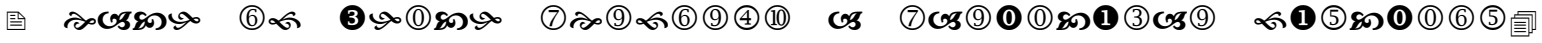
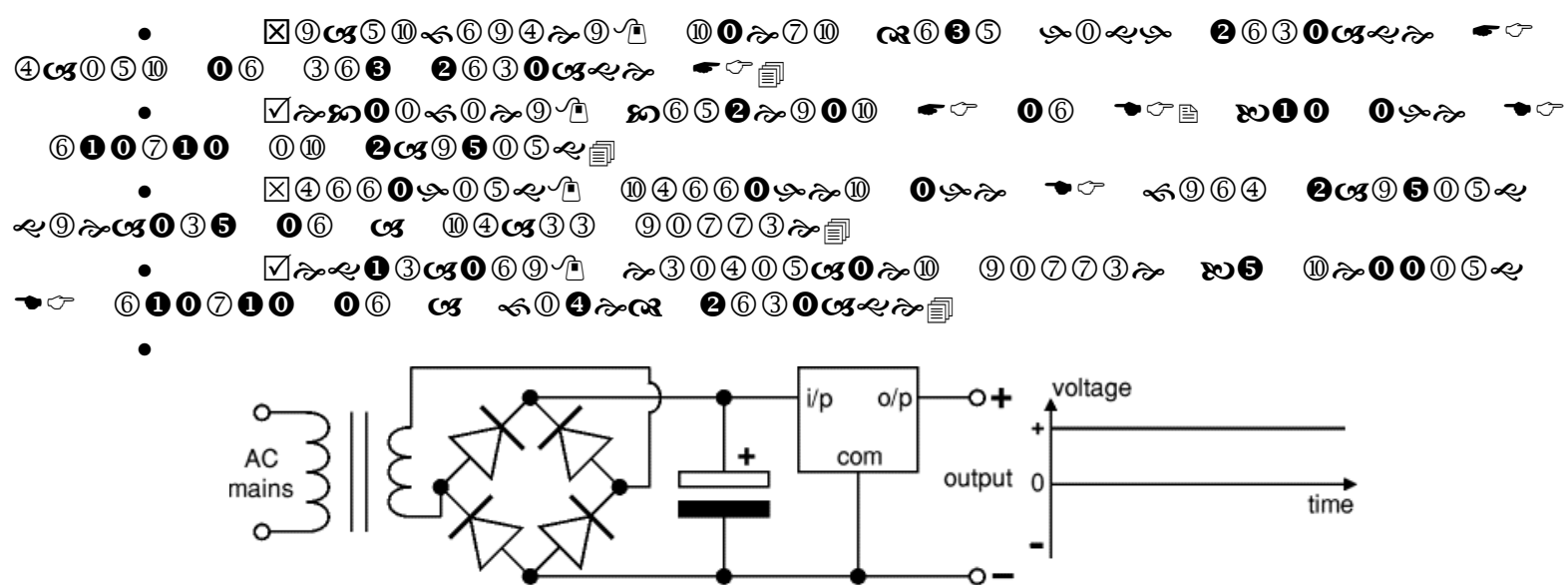

Transformer Rectifier Smoothing Regulator Output: regulated DC

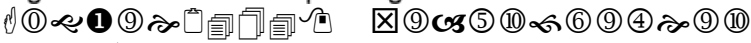

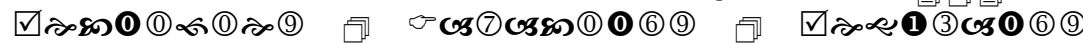

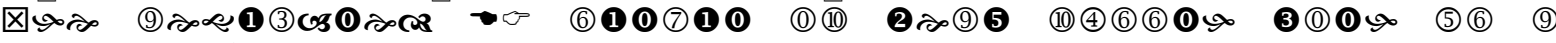

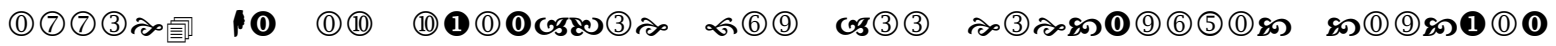
(11) 圆

\subsection{DISPLAY UNIT:}

the display unit consists of $16^{*} 2$ liquid crstal display that dispays the position of the intruder as well as the condition of the alarm system. The lower data bus of the LCD is connected to port 2 of the microcontroller while RS and EN pins are connected to port 3_0 and port 3_1 respectively.

2.3 Key pad unit

Keypads are assembled in a matrix form as illustrated below:

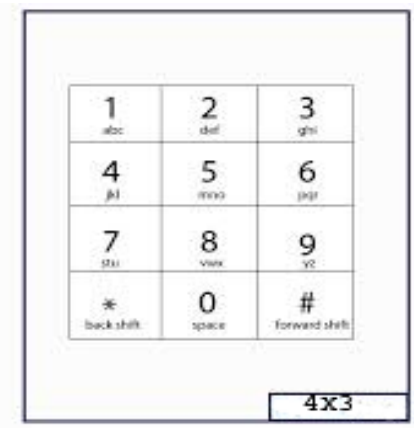

Fig $2.34 \times 3$ matrix key pad 
The above diagram shows 4x3 keypad - 12 switches in a default state (all switches open) there is no connection between the rows and columns. When the switch is pressed a connection between the switch's columns is made.

\section{KEYPAD DECODER}

Many keypads are built with an onboard decoder that scan the keypad, and if a key is pressed, returns a number identifying the key.

The decoder has 7 inputs; the $4 \mathrm{x}$ inputs are connected to the 4 keypad columns while the $\mathrm{y}$ inputs are connected to the 3 keypad rows.

How the keyboard is scanned.

The steps are

Scan row 1

Scan row 2

Scan row 3

Scanning a row is achieved applying $0 \mathrm{~V}$ to the port pin for that row and $5 \mathrm{~V}$ to the other three rows, then scanning each individual column to see if one of them is low. If it is, then the key at the junction between the current row and column being scanned is the pressed key.

Clear row 1, set other 3

Scan column 1

Scan column 2

Scan column 3

Clear row 2, set other 3

Scan column 1

Scan column 2

Scan column 3

Clear row 3, set other 3

Scan column 1

Scan column 2

Scan column 3

For example, let's say the key being pressed is key 6 . When scanning the first row, P1.0 will be cleared while the other 3 rows (P1.1, P1.2, and P1.3) are set. Since no key in this row is closed, there is no path for current through any of the pull up resistors and all 3 columns (on P1.4 to P1.6) are high. Therefore the key pressed was not found while scanning row 1.

Scanning row 2 (with 6 still closed) in this case, column 3 is connected through the closed switch to row 2. Since row 2 is low, column 3 is low

The keypad is initialized in the main program all rows are cleared. Therefore when a key is pressed, since all rows are LOW, then one of the columns (the one containing the key that has been pressed) will be connected to $0 \mathrm{~V}$.

\subsection{Microcontroller unit:}

The AT89C51 is a low power, high performance cmos 8-bit microcontroller with 4Kbytes of flash programmable and erasable read only memory (PEROM). The device is manufactured using Atmel`s high density nonvolatile memory technology and is compatible with the industry standard MCS-51 instruction set and pinout. The on-chip flash allows the program memory to be reprogrammed in system or by a conventional nonvolatile memory programmer. By combining a versatile 8-bit CPU with flash on a monolithic chip, the Atmel AT89C51 is a powerful microcomputer, which provides a highly flexible and cost effective solution to many embedded control application.The AT89C51 is designed with static logic for operation down to zero frequency and support two software selectable power saving modes. The idle mode stops the CPU while allowing the RAM, timer/counters, serial port and interrupt system to continue functioning. The power down mode saves the RAM contents but freezes the oscillator disabling all other chip functions until the next hardware reset. 


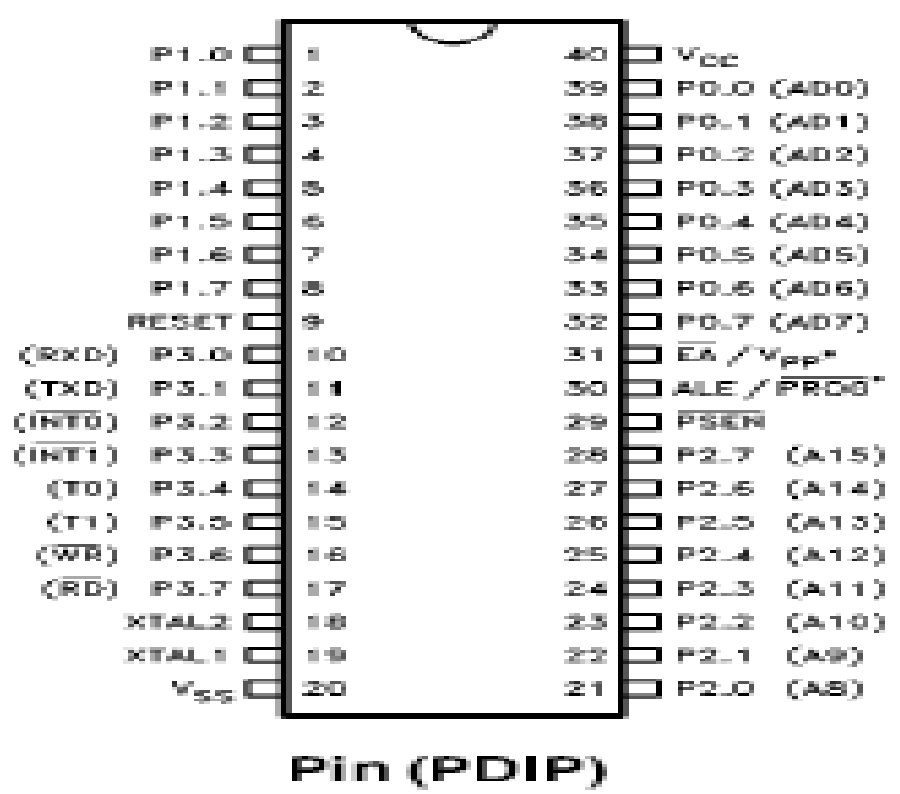

Fig. 3.22: Pin configuration of AT89S51

\section{Features of AT89S51}

- $\quad$ Programmable serial channel

- $\quad$ Compatible with MCS-51TM product

- 4Kbytes of in-system Reprogrammable flash memory - Endurance: 1,000 write/Erase cycles

- $\quad$ Fully static operation: $0 \mathrm{~Hz}$ to $24 \mathrm{MHz}$

- $\quad$ Three-level program memory lock

- 128 x 8-bit internal RAM

- 32 programmable I/O lines

- $\quad$ Two 16-bit timer/counters

- $\quad$ Six interrupt sources

- $\quad$ Low-power idle and power-down modes.

\subsection{Alarm unit}

This consists of an LED, which connects from the P0.7 of the micro controller, which in turn connects to $1 \mathrm{~K} \Omega$ resistor, which finally connects to the VCC. The LED indicates on the alarm is set to slow.

P3.7 of the micro controller connects to the resistor $(1 \mathrm{~K} \Omega)$, through the base of the transistor (NPN C945) and the collector to the ground, while the emitter terminal is connected to the negative terminal of the alarm speaker, and the positive to the Vcc. The remaining terminal of the resistor to Vcc, as shown in the figure 3.4

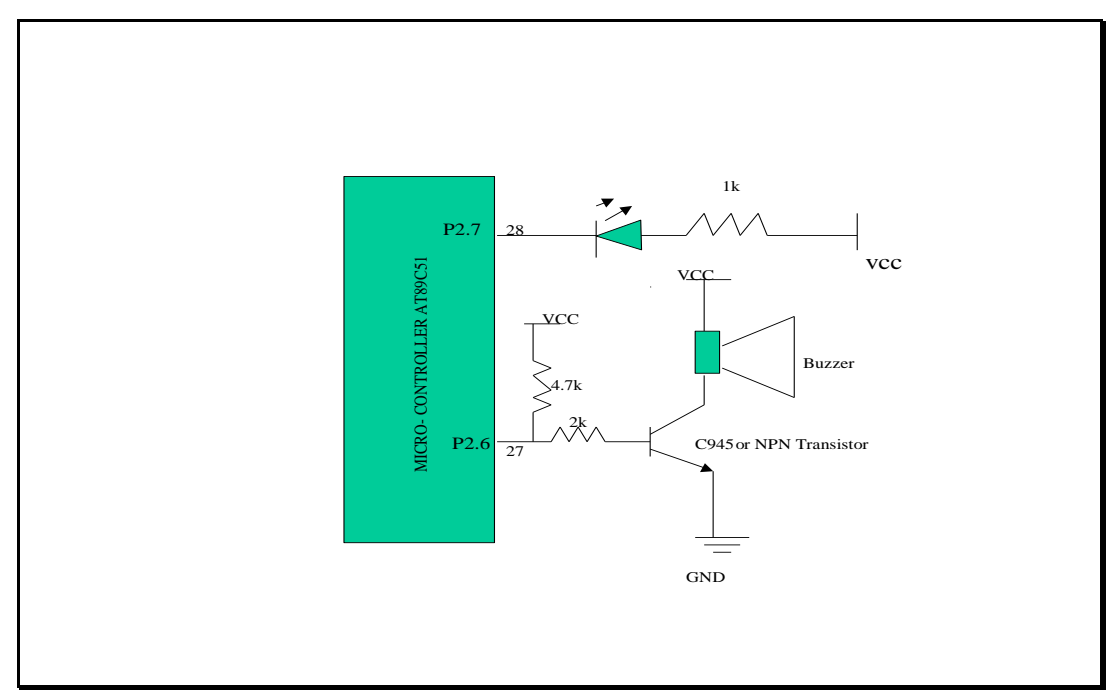

Fig. 3.4 Micro controller interface with alarm unit 


\subsubsection{Door control segment:}

The door control segment consists of an H-Bridge driver IC that guarantee's the clockwise and the anti clockwise movement of the electric motor attached to the door system. The H-Bridge driver IC L293D is designed to control 2 DC motors. There are 2 input and 2 output pins for each motor. The 2 pairs of input determine the behavior of electric motor attached to it. The chip has two enable pins that must be high $(+5 \mathrm{~V})$ for operation.

The behavior of motor for various input conditions are as follows:

\begin{tabular}{|l|l|l|}
\hline & \multicolumn{1}{|c|}{ A } & \multicolumn{1}{c|}{ B } \\
\hline Stop & Low & Low \\
\hline Clockwise & Low & High \\
\hline Anti clockwise & High & Low \\
\hline Stop & High & High \\
\hline
\end{tabular}

Table 3.5: H-bridge motor control

\section{Design Process:}

A micro-controller based project design process is characterized by the following;

* Definition of task

* Requirements

* Factor that influence choice

In defining a task, every design comes from an idea or a problem that requires a solution. Questions may be generated on what exactly that is required to be achieved and the feasibility of the ideas as regards to the implementation.

If these questions are analyzed critically with tangible solutions to the problem, a development of this idea into a reality is the next step.

Requirements for design process have to be considered once an idea has been established. The need to determine whether or not the idea requires a PC or not, depending on the complexity of the circuitry, or whether the circuits to be designed needs to make a complex decision or deal with complex data.

The compare these factors with topic with IC's (AND gates) which two inputs when high logic changes outputs. Preferably, a micro-controller will be the best option based on the circuits to be designed with less hardware connections and flexibility. Writing a program that performs a desired function accesses the ability of the micro-controller. However, it came to our thought that among all the components used here the one that consumes the highest power is the Light Emitting Diodes (LEDs), which need as much as 15-volt to glow and draw as maximum as 2-ampere of current. As a result if this, I decided to use 15-volts, 2-ampere rated transformer for this design. After the voltage is stepped down 15-volts using a transformer, a full wave rectifier circuit was designed using four Diodes (IN4001). This value of Diodes is used here because from the specification of voltage/Diode rectifying data book, this value is adequate for lower voltages, say 0-24 volt. The load current of the rectifier is given as follows;

$\mathrm{Idc}=21 \mathrm{~m} / 3.1$

When $1 \mathrm{~m}=$ maximum current $=2 \mathrm{amp}$

$\mathrm{Idc}=$ load current which is in dc form after recification.

Hence idc $=2 * 3 / 3.14=4 / 3.14$, idc $=1.27$ amps

Therefore $1.27 \mathrm{amps}$ is the maximum load current that can be drawn in the whole system. Also it is known that after rectification, the same voltage (15-volts) continues to flow into the filter. As a result the load voltages 15volts

Thus Vdc $=$ Load voltage $=15$ volts.

And $\mathrm{Vdc}=21 \mathrm{~m} * \mathrm{RL} / 3.14$

$\mathrm{RL}=$ Load resistance

$\mathrm{RL}=47.13 / 4$

$\mathrm{RL}=11.78 * 3.14=1.78$

From this, it is obvious that $\mathrm{V}=\mathrm{IR}=1.27 * 1.27$ 
III. System Block Diagram

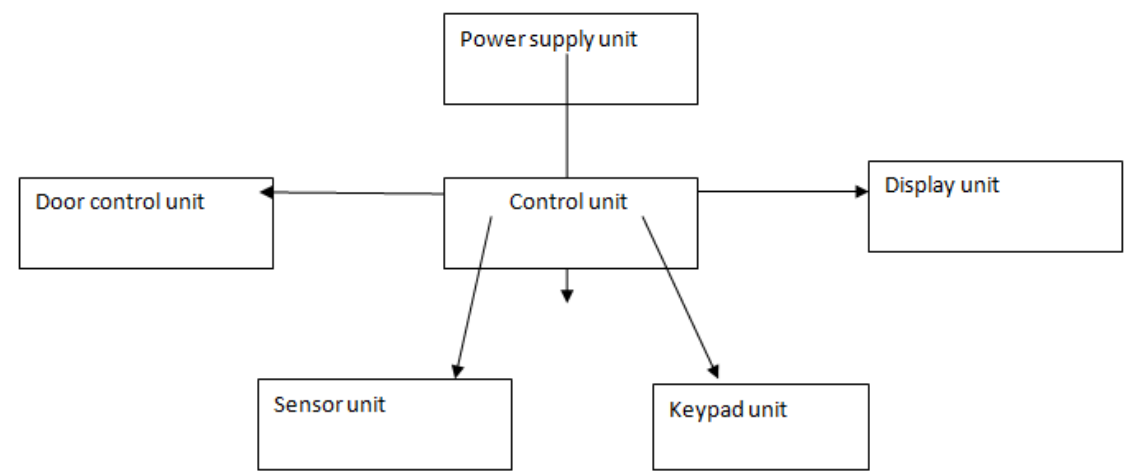

Figure 4.1 Block Diagram of Microcontroller Based code based locking system

\subsection{System Flowchart}

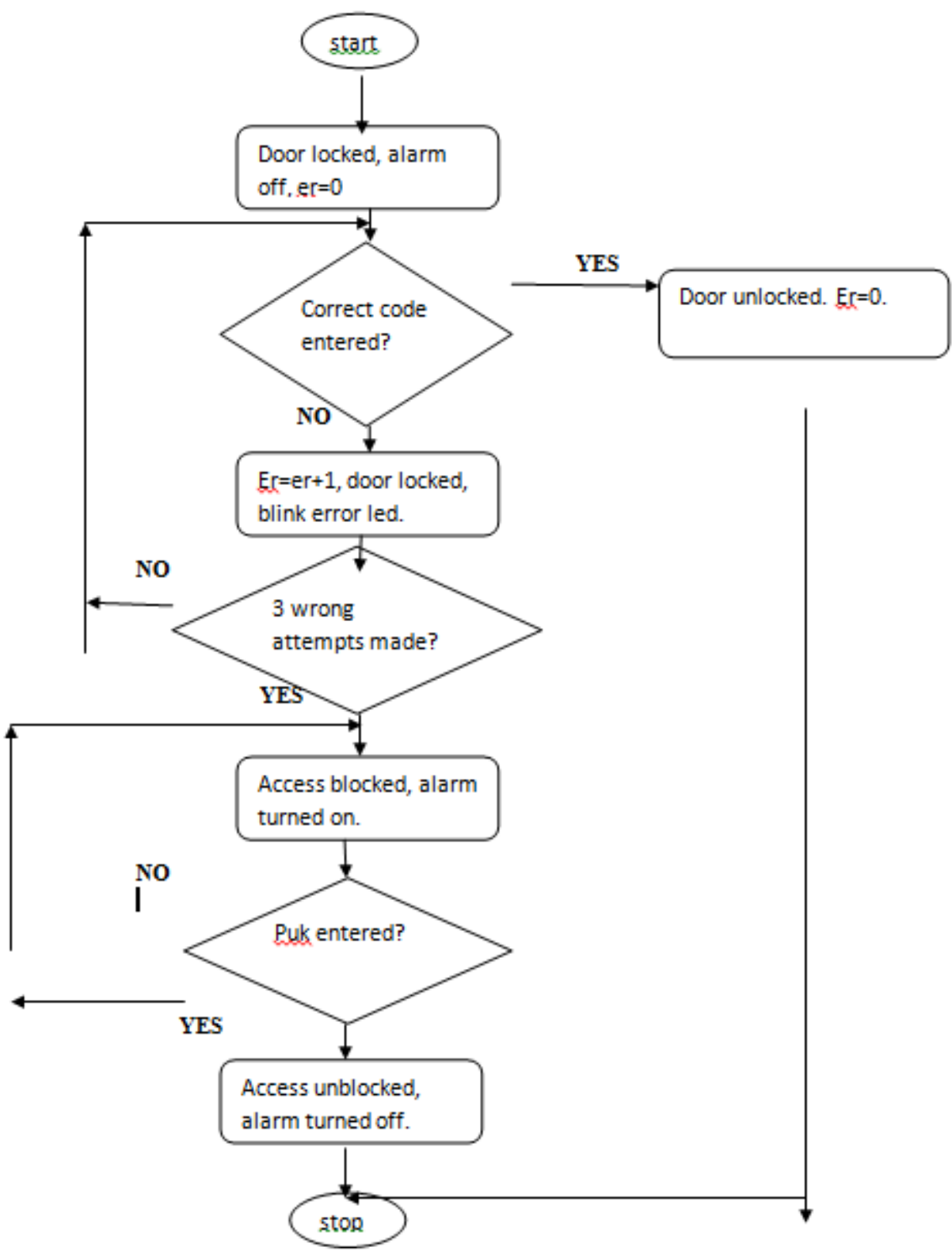

Fig 4.2 system flow chat 


\subsection{CIRCUIT DIAGRAM}

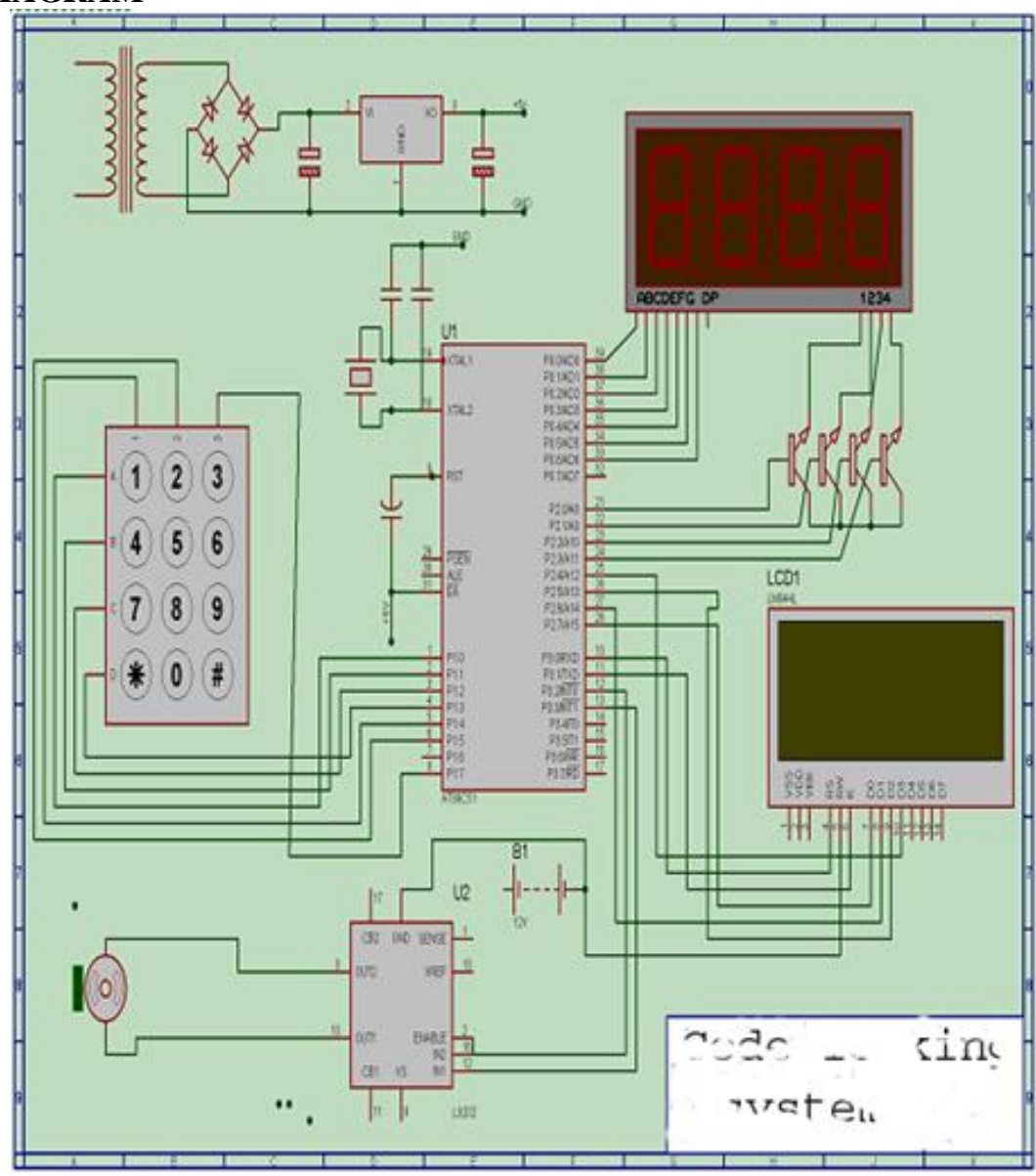

Fig 4.3 circuit diagram

IV. Software Design

Software, are computer programs; instructions that cause the hardware, the machines to do work. Software as a whole can be divided into a number of categories based on the type of work done. The two primary software categories are operating systems (system software), which control the workings of the computer, and application software, which addresses the multitude of tasks for which people use computers.

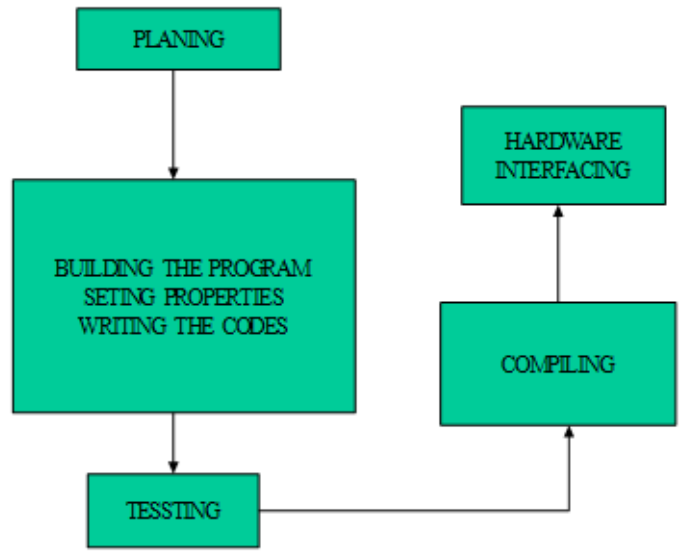

Fig .5.1 Software Design 


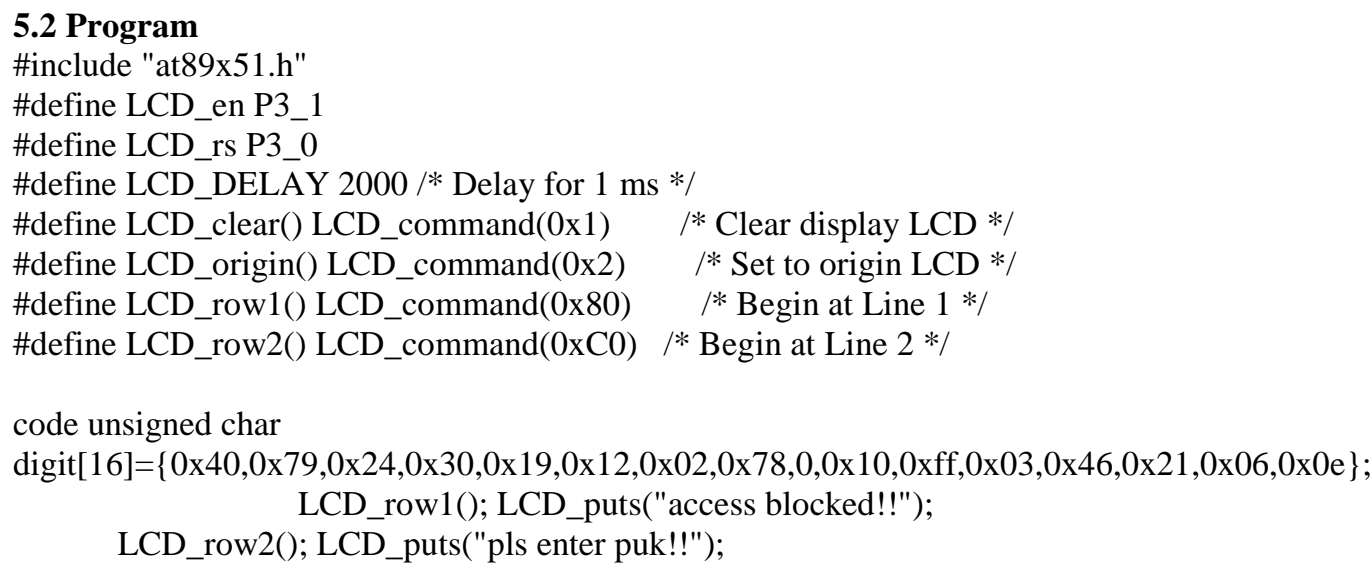

\section{Conclusion}

This paper microcontroller based code locking system with alarmhas successfully presented a functional and an easy way to combat crime and theft using a low cost device, and can be remotely applied everywhere security is needed.

\section{REFERENCES}

[1]. Belone Schilling, Electronic Circuits: Discrete and Integrated, McGraw-Hill, New York,1979.

[2]. B.L Theraja and A.K Theraja, A Textbook on Electrical Technology, 2003, 23 ${ }^{\text {rd }}$ Edition, Pp 1887 - Pp 1888.

[3]. Paul Horowitz and Windfield Hill, The Art of Electronics, 1989, $2^{\text {nd }}$ Edition, Pp 7- Pp 8, Pp 55 - Pp 58, Pp 614 -Pp 622.

[4]. Atmel Corporation Data Sheet on AT89C51, 0285D-B-12/97

[5]. NTE Electronics Inc., ECG Data Book, January 2002, $10^{\text {th }}$ Edition.

[6]. Giorgio Rizzoni, Principles of Electrical Engineering, 2003, $3^{\text {rd }}$ Edition 